\title{
Networks take the edge off parking
}

IEEE Access 6, 70172-70185 (2018)

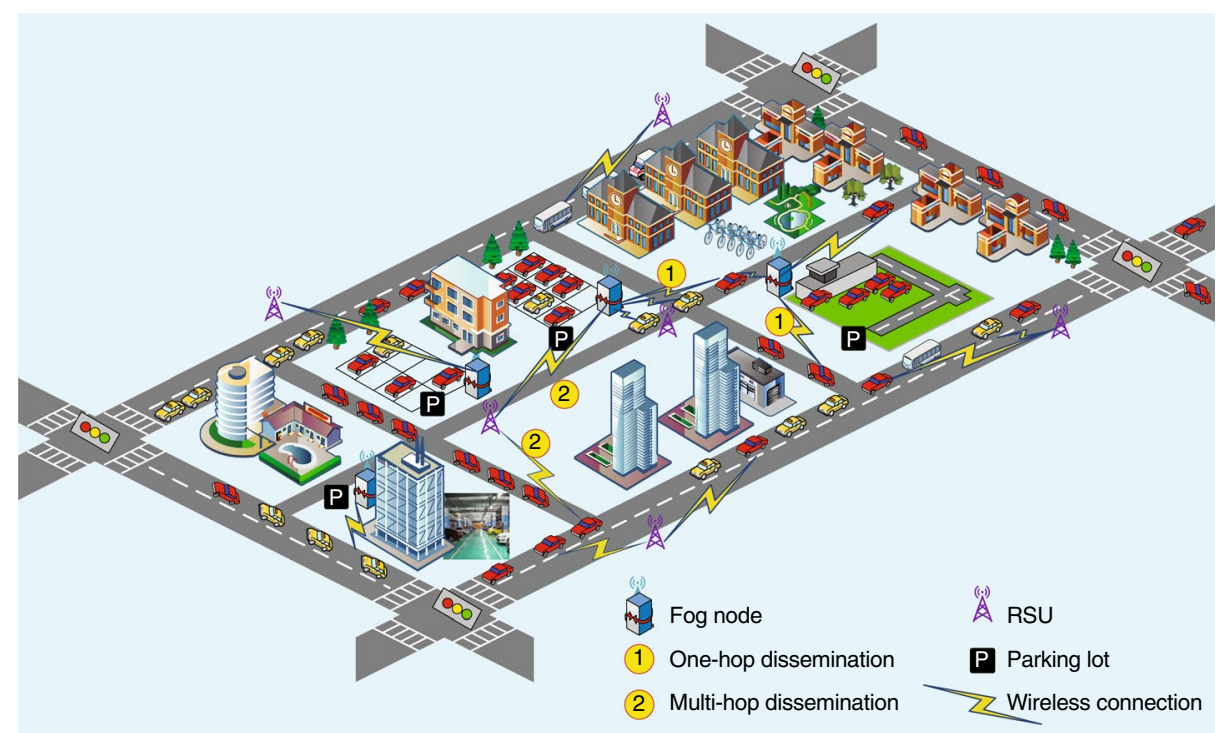

\section{Credit: IEEE}

Finding a parking space in densely populated cities can be challenging. Smart parking systems based on vehicle ad hoc networks (VANETs), which aim to deliver useful parking information to drivers in realtime, could be a solution. However, existing vehicle communication devices and roadside units (RSUs) that make up the VANET have limited computational power, and thus fail to offer real-time parking slot information and future parking space predictive capabilities. Chunsheng Zhu and colleagues now propose VANETs with cloud and fog computation capabilities to collect, analyse and share traffic and parking information to help drivers decide where to park.

The basic idea is to employ fog nodes, which are placed at parking lots and intersections, to process parking lot utilization and traffic information, and then communicate this information to other vehicles through the use of RSUs. The researchers - who are based at institutes in China, Canada, Brazil and Portugal also suggest that a remote cloud data centre could be employed to provide a wider overview of the parking area, and to enable a level of global optimization in the decision-making process. The ability to allocate parking spaces more effectively may lead to reduced driving time, which should lead to more efficient fuel use and reduced vehicular emissions.

\section{Michael Lee}

Published online: 16 January 2019 https://doi.org/10.1038/s41928-018-0199-5 